\title{
Visual Impairment as a Public Health Problem
}

\author{
YOSHIMUNE HIRATSUKA* \\ * Department of Ophthalmology, Juntendo University Faculty of Medicine, Tokyo, Japan
}

\begin{abstract}
$80 \%$ of all visual impairment can be prevented or cured because uncorrected refractive error and cataract are two leading causes of visual impairment in the world. Half of visual impairments occur in those age 70 years and older. The social costs of visual impairment is substantial, because visual impairment places a heavy burden on individuals, families, and society, and a lot of resources are dedicated to care for individuals with visual impairment at home through informal care. With the accompanying trend of population ageing, the need to prioritise healthy ageing is increasingly recognized, and it is increasingly important to extend healthy life expectancy. Measures against visual impairment will help to extend healthy life expectancy.
\end{abstract}

Key words: adipose-derived stromal/stem cell, keratinocyte, fibroblast, collagen, epidermolysis bullosa

A loss of eyesight is frightening. $90 \%$ of information absorbed by the brain is visual, so the visual impairment is the important issue in the society.

\section{$80 \%$ of all visual impairment can be prevented or cured}

Common definition of visual impairment used world wide is based on the World Health Organization (WHO) criteria. They used to use bestcorrected visual acuity (BCVA) in the betterseeing eye for their definitions. However, in the year of 2008, WHO changed the definition of visual impairment from "best corrected" to "presenting", that is acuity with whatever refractive correction the person is currently using, because it is more appropriate as it enables uncorrected refractive error to be included as a cause of visual impairment. Now, the definition of visual impairment is as presenting visual acuity of less than 0.3 but 0.05 or better in the better-seeing eye, and blindness is as presenting visual acuity of worse than 0.05 in the better-seeing eye. Globally 32.4 million people were blind in 2010, and an additional 191 million people had moderate and severe vision impairment (low vision) in $2010^{1)}$. The number of people visually impaired from infectious diseases has reduced in the last 20 years, uncorrected refractive errors are the main cause of visual impairment; cataracts remain the leading cause of blindness in the world. Uncorrected refractive error are correctable and cataract are operable/curable, therefore $80 \%$ of all visual impairment still can be prevented or cured. Half of visual impairments occur in those age 70 years and older.

\section{The costs of visual impairment is substantial}

Visual impairment places a heavy burden on individuals, families, and society. Increasing eye disease and vision loss is often driven by an aging population and social and environmental changes. We have shown that in 2007 visual impairment affected more than 1.64 million people in Japan, or

\footnotetext{
Yoshimune Hiratsuka

Department of Ophthalmology, Juntendo University Faculty of Medicine

2-1-1 Hongo, Bunkyo-ku, Tokyo 113-8421, Japan

TEL: +81-3-3813-3111 E-mail: yoshi-h@tkf.att.ne.jp

〔Received Jan. 5, 2017〕〔Accepted Feb. 21, 2017〕
}

Copyright (C) 2017 The Juntendo Medical Society. This is an open access article distributed under the terms of Creative Commons Attribution License (CC BY), which permits unrestricted use, distribution, and reproduction in any medium, provided the original source is properly credited. doi: $10.14789 / \mathrm{jmj} .63 .201$ 


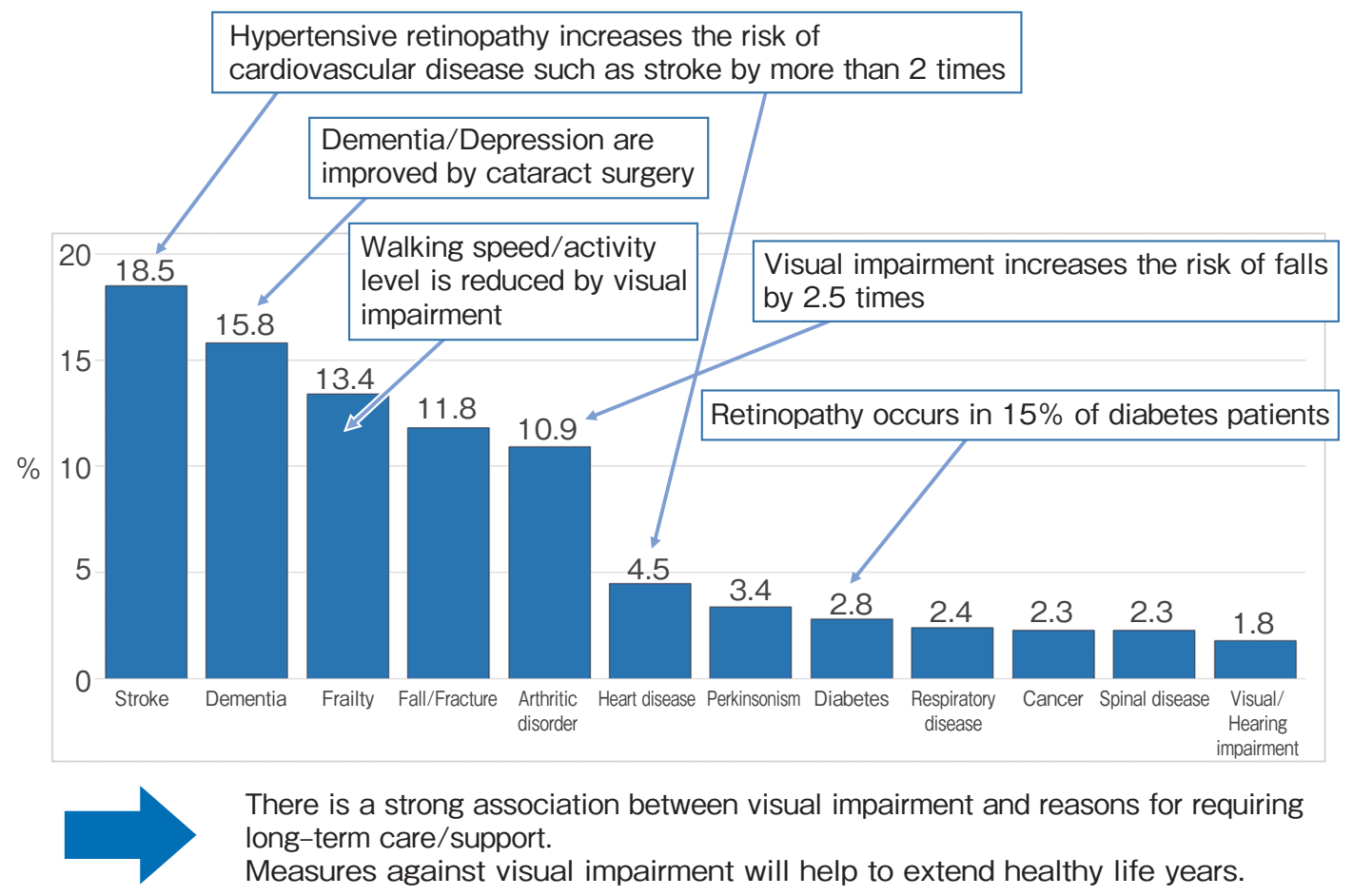

Figure-1 Reasons for requiring long-term care/support (2013 National Livelihood Survey)

$1.3 \%$ of the population ${ }^{2)}$, and cost an estimated $¥ 8,785.4$ billion [US $\$ 72.8$ billion], or $1.7 \%$ of the gross domestic product of Japan ${ }^{3)}$. These findings are consistent with other studies that also found substantial expenditures to be associated with visual impairment. Our analysis suggests that community care costs account for $50 \%$ of indirect costs of visual impairment in Japan. This suggests that substantial resources are dedicated to care for individuals with visual impairment at home through informal care.

\section{Intervention to visual impairment as a prevention of long-term care/support}

In 2014, the expectancies at birth were 81 years for males, and 87 for females in Japan. With the accompanying trend of population ageing, the need to prioritise healthy ageing is increasingly recognized, and it is increasingly important to extend healthy life expectancy. Healthy life expectancy is the number of years that a person at a given age can expect to live in good health taking into account age-specific mortality, morbidity, and functional health status. In this regard, Japan leads the world, and male and female healthy life expectancy at birth in 2010 was 71 and 76 in Japan, respectively ${ }^{4)}$. However, the number of people certified for long-term care/support need is 6,150,000 in 2016, and this increase in demand for long-term care/support poses a great challenge for the health system in Japan. The reasons for which services were needed were stroke (18.5\%), dementia (15.8\%), frailty $(13.4 \%)$, falls/fractures (11.8\%), arthritic disorders (10.9\%), and others in 2013 (Figure-1). Most of these reasons are closely related to visual impairment. For example, hypertensive retinopathy increases the risk of cardiovascular disease such as stroke by more than 2 times. Dementia or depression are improved by cataract surgery, walking speed/activity level is reduced by visual impairment, visual impairment increases the risk of falls by 2.5 times, and finally visual impairment itself is one of the reasons for requiring longterm care/support. There is a strong association between visual impairment and reasons for requiring long-term care/support. Therefore, measures against visual impairment will help to extend healthy life years, and this fact should be more widely recognized by society. 


\section{Reference}

1) Stevens GA, White RA, Flaxman SR, et al: Global prevalence of vision impairment and blindness: magnitude and temporal trends, 1990-2010. Ophthalmology, 2013; 120: 2377-2384.

2) Yamada M, Hiratsuka Y, Roberts CB, et al: Prevalence of visual impairment in the Japanese population by cause and severity and future projections. Ophthalmic Epidemiol, 2010; 17: 50-57.

3) Roberts CB, Hiratsuka Y, Yamada M, et al: Economic cost of visual impairment in Japan. Arch Ophthalmol, 2010; 128: 766-771.

4) Salomon JA, Wang H, Freeman MK, et al: Healthy life expectancy for 187 countries, 1990-2010: a systematic analysis for the Global Burden Disease Study 2010. Lancet, 2012; 380: 2144-2162. 